\title{
Myofibroblasts in Schistosomal Portal Fibrosis of Man
}

\author{
Zilton A Andrade/ ${ }^{+}$, Sylviane Guerret*, André LM Fernandes
}

Centro de Pesquisas Gonçalo Moniz-Fiocruz, Rua Valdemar Falcão 121, 40295-001 Salvador, BA, Brasil

*Laboratoire Marcel Merieux, Avenue Tony Garnier, Lyon, France

Myofibroblasts, cells with intermediate features between smooth muscle cells and fibroblasts, have been described as an important cellular component of schistosomal portal fibrosis. The origin, distribution and fate of myofibroblasts were investigated by means of light, fluorescent, immunoenzymatic and ultrastructural techniques in wedge liver biopsies from 68 patients with the hepatosplenic form of schistosomiasis. Results demonstrated that the presence of myofibroblasts varied considerably from case to case and was always related to smooth muscle cell dispersion, which occurred around mediumsized damaged portal vein branches. By sequential observation of several cases, it was evident that myofibroblasts derived by differentiation of vascular smooth muscle and gradually tended to disappear, some of them further differentiating into fibroblasts. Thus, in schistosomal pipestem fibrosis myofibroblasts appear as transient cells, focally accumulated around damaged portal vein branches, and do not seem to have by themselves any important participation in the pathogenesis of hepatosplenic schistosomiasis.

Key words: myofibroblast - schistosomiasis - pipestem fibrosis - liver

The characteristic hepatic lesion seen in patients with hepatosplenic schistosomiasis is represented by systematized portal fibrosis which appears at the cut surface of the liver as whitish plaques against a background of normal looking parenchyma, the so-called Symmers' clay pipestem fibrosis (Symmers 1904). The microscopic counterpart is also characteristic, being represented by expansion of the portal spaces by fibrosis, with total or partial destruction of the portal vein branch contrasting with the preservation of the biliary and hepatic arterial structures (Andrade et al. 1992). Ultrastructural findings have revealed another important feature: the outstanding presence of myofibroblasts, a cell-type with intermediate features of both fibroblasts and smooth muscle cells (Gabbiani 1996), that in schistosomal portal fibrosis was noted to be a prominent cell type (Grimaud \& Borojevic 1977, 1986).

Besides the capacity to synthesize extracellular matrix, myofibroblasts could play a role in the pathogenesis of portal hypertension due to their contractile properties. The myofibroblast is a prominent cell-type in abnormal scar tissue, such as occurs in Peronye's disease, Dupuytren's dis-

Supported by Pronex.

Corresponding author. Fax: +55-71-356.8787. E-mail: zilton@server01.cpqgm.fiocruz.br

Received 26 June 1998

Accepted 26 October 1998 ease, keloids and scar contractures, especially those due to burns (Gabbiani \& Majno 1972, Eddy et al. 1988, Kuhn \& McDonald 1991, Berndt et al. 1994).

These implications contribute to give the participation of myofibroblasts in hepatic schistosomiasis a special interest. However, the true significance of myofibroblasts in schistosomal portal fibrosis has not been sufficiently established. The present investigation concerns a morphological study on the presence, distribution, origin and fate of myofibroblasts in portal fibrosis due to advanced schistosomiasis in human patients.

\section{MATERIALS AND METHODS}

Wedge liver biopsies were performed upon a total of 68 patients, 53 males and 15 females, during splenectomy, accompanied or not by vascular shunts, and done as a surgical treatment to ameliorate the manifestations due to portal hypertension. The ages of the patients varied from 16 to 61 years (average 30.5). The diagnosis of hepatosplenic schistosomiasis was made on clinico-parasitological grounds in all patients. The presence of schistosomal portal fibrosis (Symmers' pipestem fibrosis), was confirmed histologically. No other major hepatic disease was present.

Histology - Liver tissue was either fixed in $\mathrm{pH}$ 7.2 phosphate buffered $10 \%$ formalin or in Bouin's fluid, followed by paraffin embedding. Sections were routinely stained with hematoxylin-eosin, sirius-red-fast green for collagen, orcein for elastic fibers, period-acid Schiff with and without previous diastase treatment and the Perls' method for iron. 
Immunohistochemistry - Formalin-fixed paraffin-embedded sections were also submitted to immunohistochemical investigation with previous thermal micro-wave treatment and application of a stretptavidin-biotin-peroxidase method, for the identification of actin, desmin, vimentin, and elastin. The following primary antibodies were used: mouse monoclonal anti-actin (Sigma, A2547, 1:200), rabbit polyclonal anti-desmin (Sigma, D8281, 1:20), mouse monoclonal anti-vimentin (Boehringer, 814318, 1:5) and rabbit polyclonal anti-elastin (Institut Pasteur, Lyon, 15011AC1, 1:50). For negative control sections, the primary antibody was replaced by normal mouse serum.

Immunofluorescence - Fresh fragments taken separately from eight samples were immersed in Tissue-Tek (Miles Inc., Elkhart, IN, USA) and immediately frozen in liquid nitrogen. Blocks were kept in air-tight plastic boxes at $-70^{\circ} \mathrm{C}$ until the moment they were sectioned in a cryostat at $-20^{\circ} \mathrm{C}$. Sections were fixed in dehydrated acetone and treated for immunofluorescence study for the identification of smooth muscle-related structures (aactin, desmin) and elastic tissue (elastin). Dilution for the primary antibodies were the same as mentioned above. Secondary fluoresceinated antibodies (Diagnostic Pasteur, rabbit anti-IgG, 74.561 and mouse anti-IgG, 74.641) were diluted at $1: 40$.

Electron microscopy - From six cases with outstanding portal fibrosis, tiny pieces of liver tissue were taken for electron microscope study. Fixation was made in $0.2 \%$ glutaraldehyde in $0.1 \mathrm{~mol} /$ 1, sodium cacodylate buffer, $\mathrm{pH} 7.4$, for $2 \mathrm{hr}$. After thorough washing in buffer, the fragments were post-fixed in $1 \%$ osmium tetroxide for $1 \mathrm{hr}$ and embedded in Epon resin (Polybed 812TM). Areas selected after examination of semi-thin sections, were submitted to ultrathin sectioning in a Reichert Ultra Cut automatic ultramicrotome with diamond knife. Sections were contrasted with uranyl acetate and lead citrate, and examined with a Zeiss EM109 electron microscope at $50 \mathrm{kV}$.

\section{RESULTS}

The characteristic fibrous expansion of the portal tracts associated with signs of portal vein damage and preservation of arterial and biliary structures was documented in all cases. Features of chronic hepatitis of variable degree were present in $65 \%$ of the cases. Variable degree of venous muscular coat dispersion was histologically observed in all the cases of this series. It varied from the conspicuous presence of many smooth muscle fibers and cells radiating from the main portal vein and "buried" within the dense portal fibrous tissue (Fig. 1) to a few remnants or the complete disappearance of the vein, with its replacement by fibrosis. Immunohistochemical and immunofluorescent data confirmed the histological findings. Dispersed smooth muscle cells were strongly stained by specific anti-a-smooth muscle actin antibodies (Fig. 2). Other fusiform connective cells, faintly expressing actin, probably myofibroblasts, also appeared in variable number, dispersed among the smooth muscle cells and shortly beyond (Fig. 3 ). Actin-positive cells were observed around damaged portal veins, but not anywhere else in the dense fibrous tissue of the portal space.

Many cell-types in the vicinity of muscular coat dispersion were positive for desmin and vimentin. In relation to the peri-vascular muscular dispersion these elongated and fusiform desmin and vimentin-positive cells were proportionally more evident while scattered actin-positive cells were becoming less and less numerous. Elastin was expressed in the same structures histologically stained by orcein. Elastic fibers appeared as continuous or fragmented membranes delimiting portal vein structures, and laying free in variable amount, in diffuse or focal distribution, within the portal fibrous tissue. A more dense concentration of orcein-stained fibers were seen associated with areas of smooth muscle cell dispersion.

Myofibroblasts were positively identified by means of the electron microscope only in area where the smooth muscle cells were dispersed, which occurred in the vicinity of damaged small or mediumsized portal vein branches. Several attempts were made to identify myofibroblasts within the dense fibrous tissue of the portal spaces away from the areas of venous changes, but without success. Collagen fibers with their typical periodicity were usually arranged in compact parallel bundles, sometimes accompanied by dark amorphous materials (elastin). Cellular elements were represented by typical fibroblasts and slender cytoplasmic prolongations, probably belonging to fibroblasts, and occasional leukocytes. Foci of collagen degradation showing rarefied areas with fragmented collagen fibers of different diameters and presence of granular electron dense material were sometimes seen.

The picture completely changed when an area situated in close proximity to a damaged small or medium-sized portal vein was examined. Then, typical dissociated, scattered smooth muscle cells (Fig. 4) and several intermediate cells with fibroblastic features, were abundant. These latter cells varied from fusiform to a rounded form and were characterized by the presence of dark submembranar densifications, presence of fewer cytoplasmic myofibrils as compared to smooth muscle cells, disclosing rough endoplasmic reticulum and a few mitochondria especially located in 


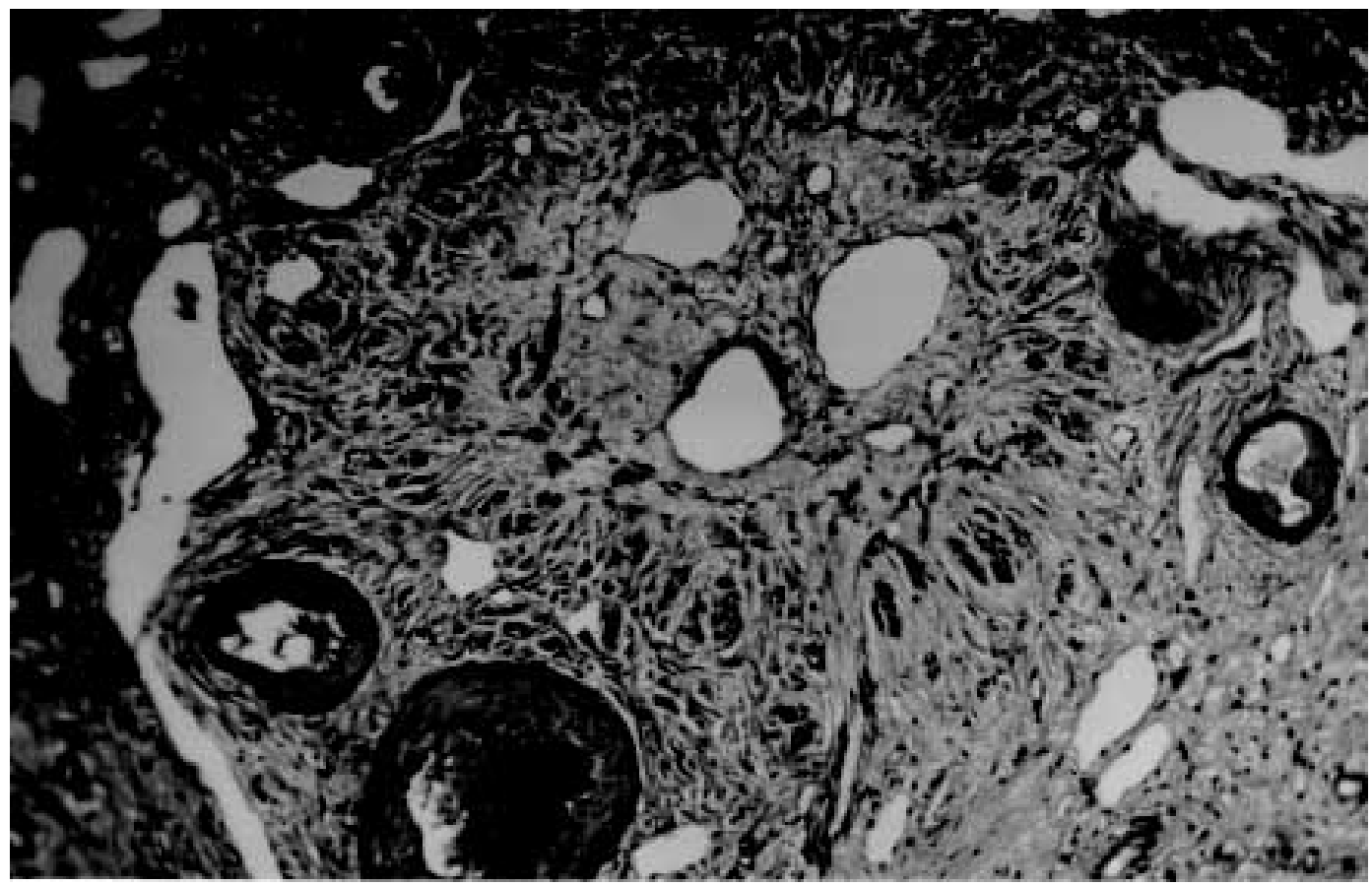

Fig 1: large branch of the portal vein suggestive of old thrombosis with organization and re-canalization, showing dispersion of smooth muscle fibers around it. The presence of such vascular lesion in a fibrotic portal space with preservation of biliary and arterial structures is almost pathognomonic of schistosomal "pipestem" fibrosis. Hematoxylin-Eosin, $200 \mathrm{X}$.

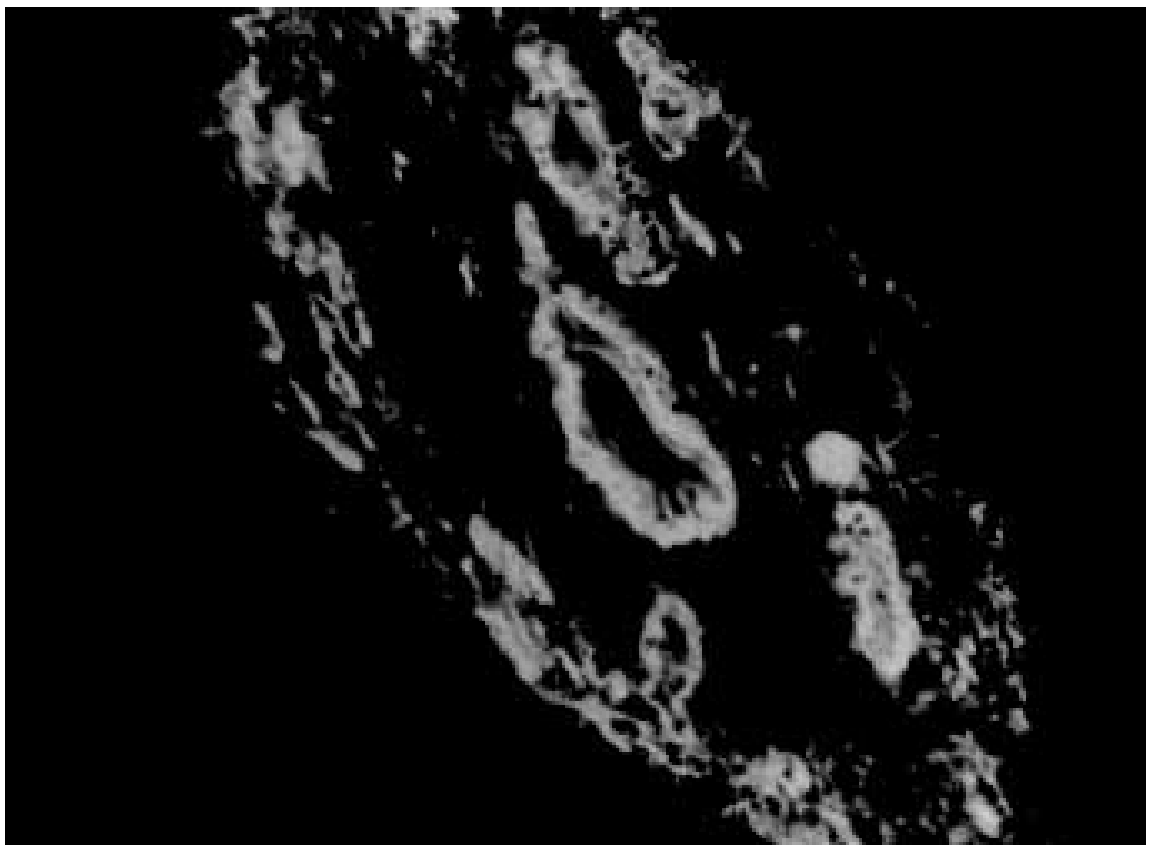

Fig 2: actin-positive fluorescense discloses the muscular coats of numerous blood vessels located in an enlarged and fibrotic portal space. Besides, many tiny structures (actin-containing cells and fibers) also appear positive. This illustrates the high degree and extension of dispersion of smooth muscle cells and associated myoid cells that can occur in some areas of schistosomal portal fibrosis. Frozen cryostat section treated with anti-actin antibodies, 200X. 


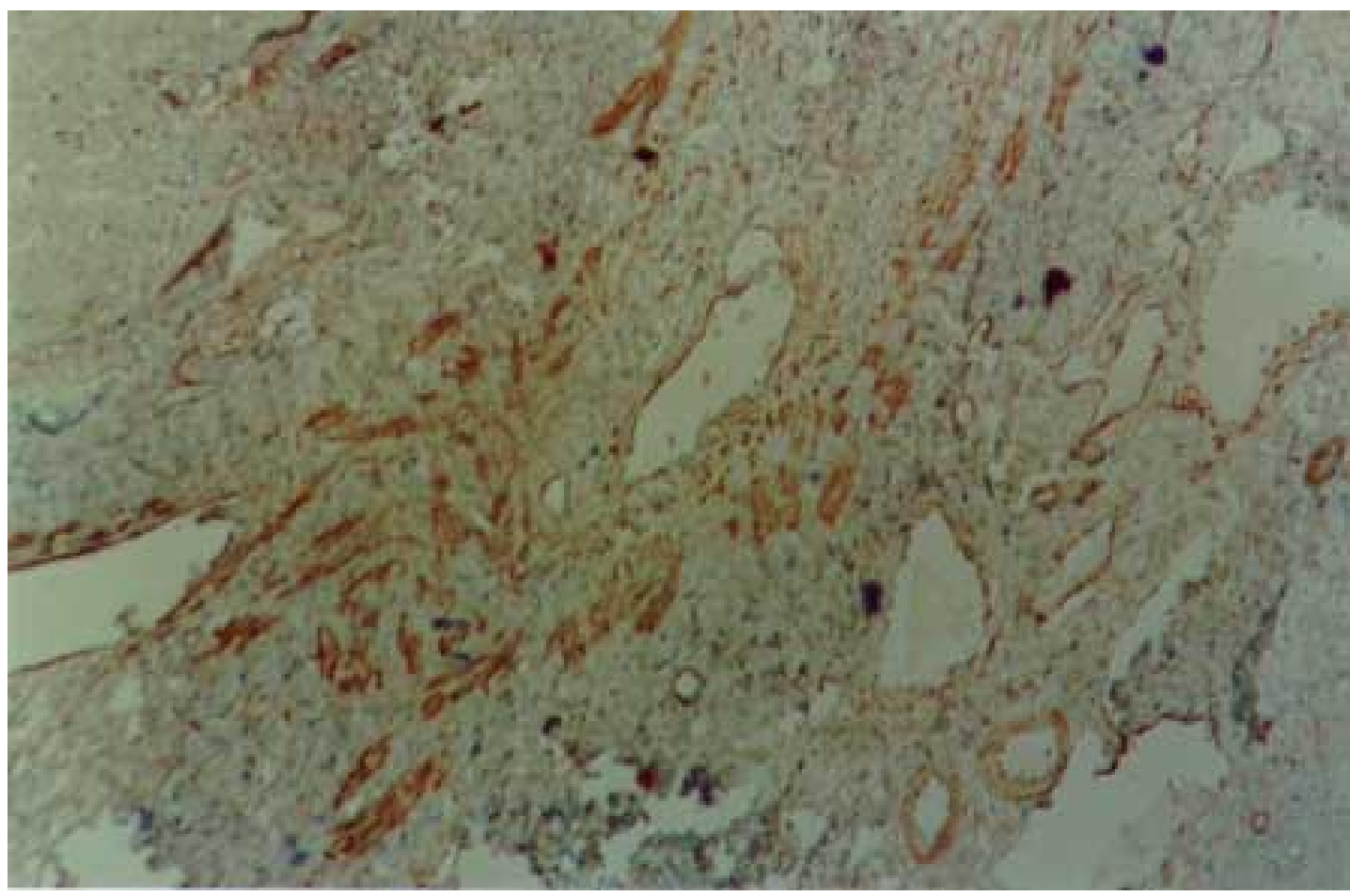

Fig 3: dispersion of actin-positive cells around a damaged branch of the portal vein. There are elongated cells of different sizes, exhibiting different degrees of staining, suggestive of transformation and also disappearance of some dispersed smooth muscle cells. Streptavidin-biotin peroxidase method, $200 \mathrm{X}$.

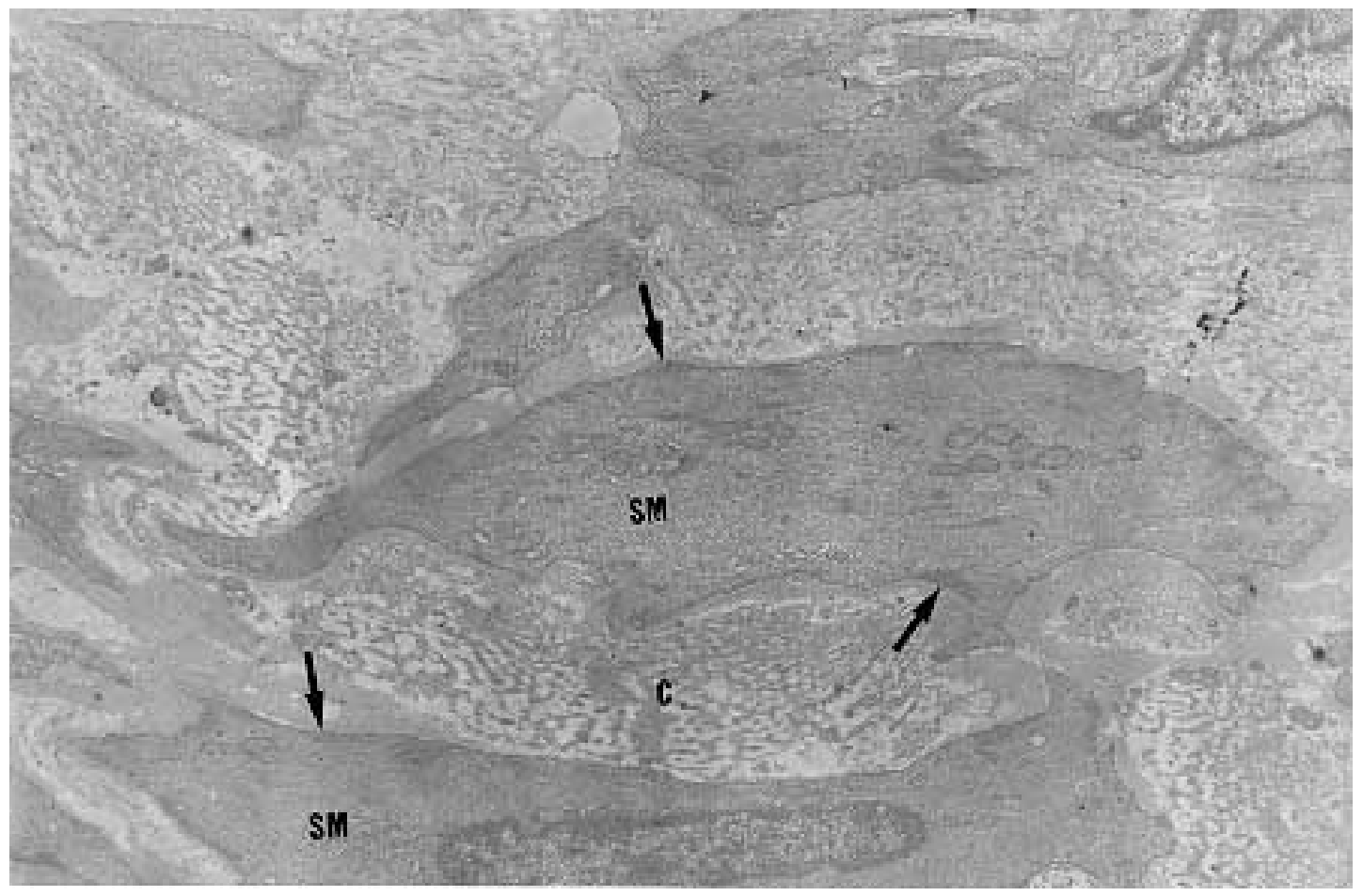

Fig: 4: typical smooth muscle cells (SM), with abundant parallel myofibrils in the cytoplasm and dark sub-membranar areas of fibril condensation (arrows) dispersed within a matrix, which exhibits numerous collagen fibers (C). Electron micrograph, 7,000 X. 
a clear perinuclear area. They appeared dispersed in several directions and isolated in the middle of collagen fibers, but when in contact with other similar cells, they frequently exhibited points of intimate cellular adhesion (Fig. 5). At some distance from the vessel wall the dispersed smooth muscle cells appeared more or less modified and sometimes assumed the features of typical myofibroblasts. Further out the myofibroblasts gradually diminished in numbers while typical fibroblasts were more frequently seen. There frequently appeared to be a transition between myofibroblasts and fibroblasts within these areas (Fig. 6).

\section{DISCUSSION}

Results from several studies have indicated that myofibroblasts are transient cells in which stress filaments (a-actin) are expressed in connective-tissue cells in response to mechanical traction or contraction (Gabbiani et al. 1972, Gabbiani 1996). In wound healing myofibroblasts usually disappear after some time through apoptosis (Gabbiani 1996), but in other circumstances they can probably revert to smooth muscle cells and/or fibroblasts.
However, as far as human schistosomal hepatic fibrosis is concerned, the only previous studies made on this subject indicate that myofibroblasts may have a more lasting and decisive presence (Grimaud \& Borojevic 1977, 1986). Based on ultrastructural findings, these authors suggested that severe hepatic lesions in schistosomiasis do not depend on the parasite eggs, but rather on a series of factors, culminating in vascular damage, myofibroblast proliferation and the production of excessive amount of extracellular matrix by these hyperplastic cellular elements. Present light, ultrastructural, immunoenzymatic and immunofluorescent microscopic findings reveal that the main portal vein branch within the expanded portal space is often completely replaced by fibrosis, leaving a few or no traces of the muscular coat "buried" into the fibrous tissue. When remnants of the vascular muscular coat are present, their amount varies considerably from case to case or even in a same case. Numerous bundles of smooth muscle may appear in a section and only of few traces of actinpositive fibrils are detected in another. Therefore, observation of multiple sections are in keeping with the interpretation that myofibroblasts are tran-

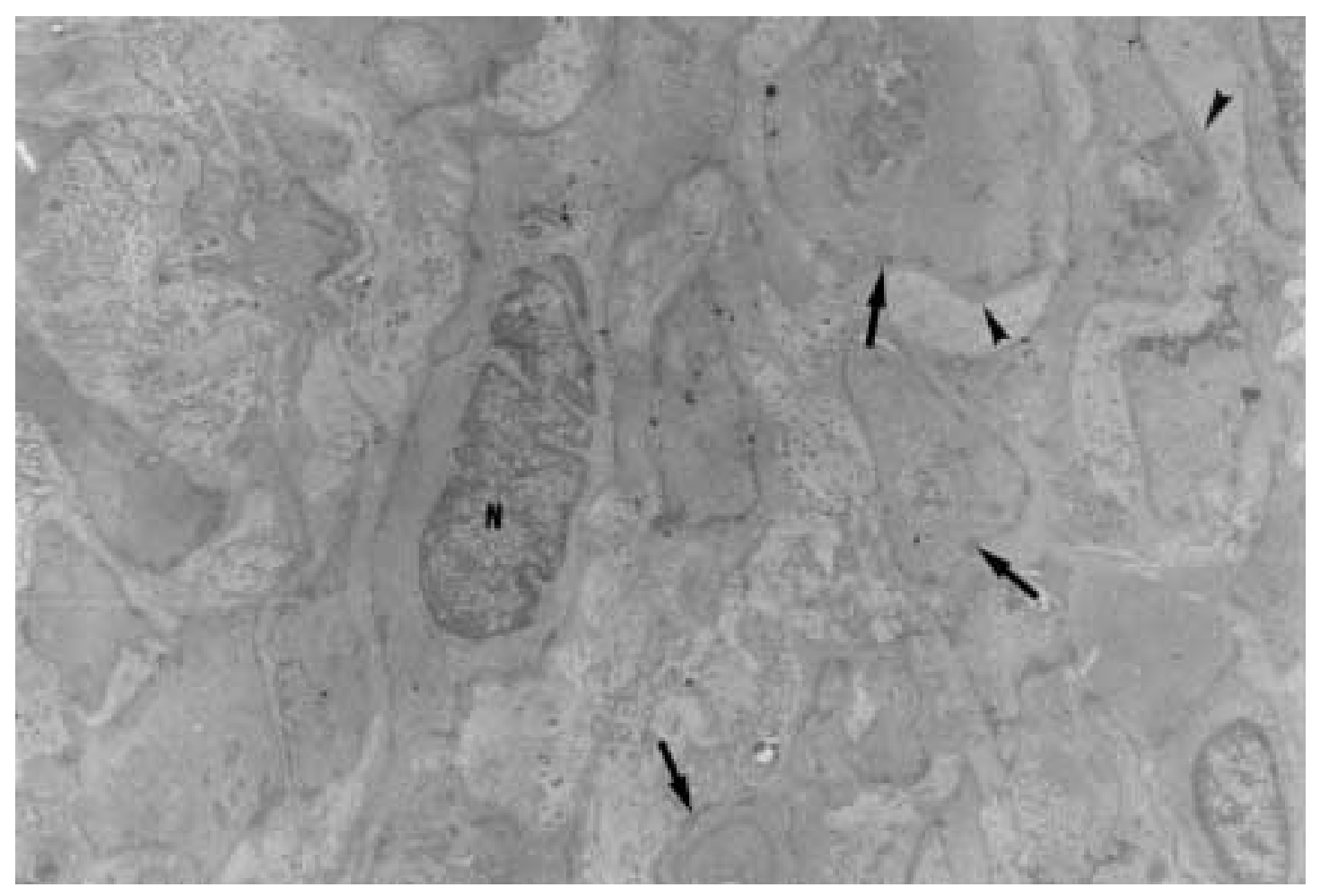

Fig 5: smooth muscle cells dispersed in a collagenous matrix and showing transformation toward a myofibroblast phenotype. The myoid cells are more round than elongated, the cytoplasmic myofibrils are less conspicous and the sub-membranous densities more evident (arrows). Some of them appear surrounded by a thin layer of an amorphous, basement membrane-like material (arrow heads). The nucleus of one of the cells (N) appears lobulated, as is commonly seen with myofibroblasts. Electron micrograph, $4,400 \mathrm{X}$. 


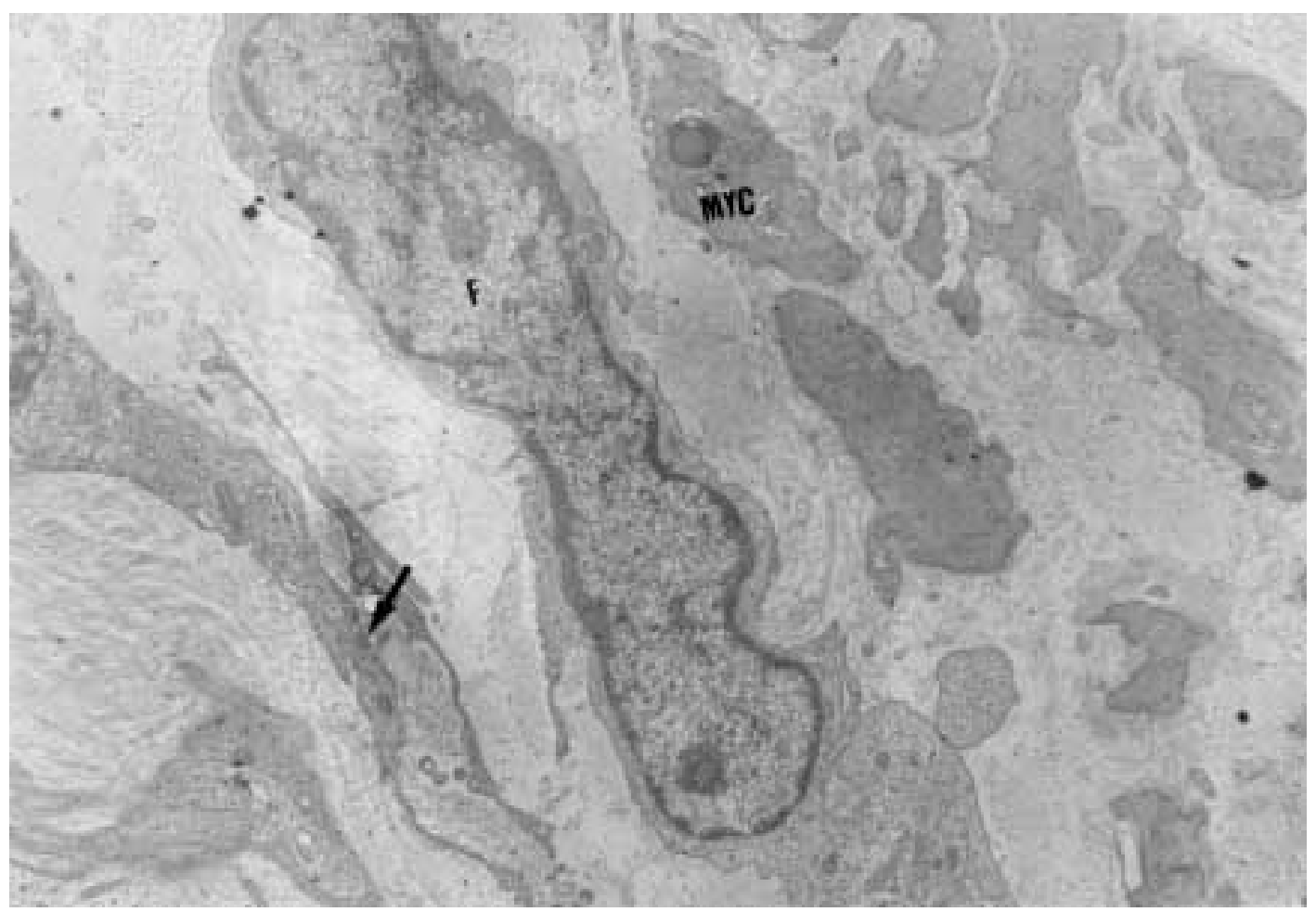

Fig 6: zone of muscular dispersion near a portal vein branch. The micrograph shows an area at the periphery of the vascular change, where one can see a typical fibroblast (F), another fibroblast exhibiting a few sub-membranous densities (arrow) and scattered myoid cells (MYC) showing myofibroblastic features (less cytoplasmic myofibrils than in a smooth muscle cell and prominent sub-membranar densifications). Electron micrograph, 3,000 X.

sient cells, most of them rapidly disappearing, some exhibiting signs of differentiation into fibroblasts. No ultrastructural evidence of apoptosis in dispersed smooth muscle cells or in myofibroblasts was seen, at least as an outstanding finding, but disappearance through apoptosis cannot be ruled out. Probably this sequence of events seen in portal tracts is far from being peculiar to schistosomiasis. As a matter of fact, such a sequence can be even experimentally demonstrated in vascular lesions during the evolution of venous grafts (Shi et al. 1996, O'Brien et al. 1997).

Experimental findings in mice have shown that myofibroblasts derived from fat-storing cells participate in the formation of periovular granulomas in portal spaces (Barbosa Jr et al. 1993, Boloukhere et al. 1993). If the same holds true for human pathology, has not been determined, but during the present study the presence of myofibroblasts (left by healed granulomas?) in the dense portal fibrous tissue, away from peri-vascular muscular coat dispersion, was not observed. Therefore, schistosomal portal fibrosis does not have the peculiar morphology of abnormal scars and myofibroblasts, transiently appearing around damaged portal vein branches, do not exhibit evidence indicating a peculiar and important participation in the pathogenesis of pipestem fibrosis.

\section{REFERENCES}

Andrade ZA, Peixoto E, Guerret S, Grimaud JA 1992. Hepatic connective tissue changes in hepatosplenic schistosomiasis. Hum Pathol 23: 566-573.

Barbosa Jr AA, Pfeifer U, Andrade ZA 1993. Role of fat-storing cells in schistosomal hepatic fibrosis of mice. Virchows Archiv B Cell Pathology 64: 91-96.

Berndt A, Kosmehl H, Katenkamp D, Tauchmann V 1994. Appearance of the myofibroblast phenotype in Dupuytren's disease is associated with a fibronectin, laminin, collagen type IV and tenascin extracellular matrix. Pathobiology 62: 55-58.

Boloukhere M, Baldo-Correa E, Borojevic R 1993. Experimental schistosomiasis mansoni: characterization of connective tissue cells in hepatic periovular granulomas. J Submicrosc Cytol 25: 505-517.

Darby I, Skalli O, Gabbiani G. 1990. a-smooth muscle actin is transiently expressed in myofibroblasts during experimental wound healing. Lab Invest 63: 21-29.

Eddy RJ, Petro JÁ, Tomasek JJ 1988. Evidence for the non-muscle nature of the myofibroblasts of granu- 
lation tissue and hypertrophic scar. An immunofluorescence stydy. Am J Pathol 130: 252-260.

Gabbiani G 1996. The cellular derivation and the life span of the myofibroblast. Pathol Res Pract 192: 708-711.

Gabbiani G, Majno G 1972. Dupuytren's contracture: fibroblast contraction? An ultrastructural study. Am J Pathol 66: 131-146.

Gabbiani G, Hirschel BJ, Ryan GB, Statkov PR, Majno $\mathrm{G}$ 1972. Granulation tissue as a contratile organ. $J$ Exp Med 135: 719-734.

Grimaud J-A, Borojevic R 1977. Myofibroblasts in hepatic schistosomal fibrosis. Experiencia 33: 890-892.

Grimaud J-A, Borojevic R 1986. Portal fibrosis: intrahepatic portal vein pathology in chronic human schistosomiasis mansoni. J Submicrosc Cytol 18: 783-793.
Kuhn C, McDonald JA 1991. The roles of the myofibroblasts in idiopathic pulmonary fibrosis. Ultrastrucctural and immunohistochemical features of sites of active extracellular matrix synthesis. Am J Pathol 138: 1257-1265.

O' Brien JE, Shi Y, Fard A, Buer T, Zalewski A, Mannion JD 1997. Wound healing around and within saphenous vein bypass grafts. J Thorac Cardiovasc Surg 114: 38-45.

Shi Y, O'Brien JE, Fard A, Mannion JD, Wang D, Zalewski A 1996. Adventitial myofibroblasts contribute to neointimal formation in injured porcine coronary arteries. Circulation 94: 1655-1664.

Symmers WStC 1904. Note on a new form of liver cirrhosis due to the presence of the ova of Bilharzia haematobia. J Pathol Bacteriol 9: 237-239. 
Myofibroblasts and Schistosomiasis - Zilton A Andrade et al. 